\title{
EI Pensamiento Crítico Como Estrategia Didáctica Musical En La Educación Emancipatoria Del Alumno En Educación Primaria
}

\author{
Luis del Barrio Aranda, (PhD) \\ Facultad de Educación, Universidad de Zaragoza, España
}

Doi: 10.19044/esj.2017.v13n35p37 URL:http://dx.doi.org/10.19044/esj.2017.v13n35p37

\begin{abstract}
Our report is concerned with the study of critical thought in musical education as a capacity which encourages the intellectual autonomy of students in primary education. After a theoretical review, based on the conceptualization and the description of the features that identify it, we provide a practical argument for its educational consideration with the development of a working with two groups of sixth grade of primary education, in which students adopt an active role becoming an element of reflection, analysis and construction of learning the musical activity. The conclusion of the study allows us to demonstrate that the promotion of reflective and critical thinking in a system of learning tasks, from cooperative work stimulates the natural development of the communication and autonomy as fundamental skills in metacognitive learning music.
\end{abstract}

Keywords: Creativity, critical thinking, musical education

\section{Resumen}

Nuestro artículo se refiere al estudio del pensamiento crítico en la educación musical como aquella capacidad que favorece la autonomía intelectual del alumno de Educación Primaria. Tras una revisión teórica, fundamentada en la conceptualización $\mathrm{y}$ en la descripción de las características que lo identifican, proporcionamos un argumento práctico a su consideración educativa con el desarrollo de un trabajo con dos grupos de sexto grado de Educación Primaria, en el que los estudiantes adoptan un rol activo convirtiendo la actividad musical en un elemento de reflexión, análisis y construcción del aprendizaje. La conclusión del estudio nos permite evidenciar que la promoción del pensamiento reflexivo y crítico en un sistema de aprendizaje por tareas, desde el trabajo cooperativo estimula el desarrollo natural de la comunicación y la autonomía como destrezas fundamentales en el aprendizaje musical metacognitivo. 
Palabras-clave: Creatividad, pensamiento crítico, educación musical

\section{Introducción}

La Conferencia Mundial sobre la Educación Superior celebrada por la UNESCO ya declaró en París (1998) que la sociedad del s. XXI sería una sociedad cognitiva, en la que el capital se iba a convertir cada vez más en un capital de conocimientos avanzados y de competencias para resolver problemas o crear soluciones nuevas. La adecuación de la educación a esta nuestra sociedad del conocimiento, en un mundo global y cambiante, sitúa al docente como el principal protagonista del cambio educativo y requiere su atención al estudio y la aplicación de nuevos conceptos, modos y estrategias educativas que faciliten el aprendizaje crítico, promuevan el pensamiento multidimensional y valoren la actitud creativa como destrezas formativas fundamentales en la educación del aprendizaje metacognitivo. Por ello, los nuevos modelos educativos además de integrar la transmisión de conocimientos, deben facilitar el desarrollo de experiencias en las que el alumno aprenda a utilizar la información para lograr conocimientos nuevos, aprenda a tomar decisiones y a resolver problemas de manera autónoma. Uno de los aspectos clave para lograr este propósito es la educación de la capacidad de aprender a aprender, como medio para la adquisición de una autonomía intelectual (Jones e Idol, 1990). En este sentido, la escuela debe incluir el trabajo de habilidades de pensamiento y su práctica mediante actividades cotidianas que contribuyan a lograr su transferencia (Sáiz y Rivas, 2008; Guzmán y Escobedo, 2006). Los responsables de la educación deben ayudar a los estudiantes a desarrollar destrezas cognitivas y organizativas del conocimiento que favorezcan su aplicación en diferentes campos de acción e integren la educación de un pensamiento de orden superior (Lipman, 1998) entendido como un pensamiento rico conceptualmente, coherentemente organizado y persistentemente exploratorio, cuyos rasgos pueden entenderse como aquellas metas a las que el pensamiento de este tipo siempre tiende a alcanzar. Una fusión entre el pensamiento crítico y creativo. Por todo ello, una de las principales cuestiones de investigadores y docentes es pensar cómo el pensamiento crítico puede ser implementado en la vida cotidiana de aula y en el entorno educativo.

\section{Pensamiento crítico y educación musical}

De acuerdo con Mertes (1991) el pensamiento crítico es aquel proceso consciente y deliberado que se utiliza para interpretar y evaluar la información y las experiencias, mediante el uso de un conjunto de actitudes y habilidades reflexivas que cuestionan las creencias y las acciones. Es decir, 
el tipo de pensamiento que aplicamos en la resolución de problemas, el cálculo de probabilidades o la formulación de inferencias en la toma de decisiones (Halpern, 1998). Desde una dimensión psicológica el pensamiento crítico integra componentes cognitivos y autorregulatorios que implican habilidades como la comprensión, la deducción, la categorización y la emisión de juicios. En su constitución y según Ennis (1985, 2011), el pensamiento crítico está formado por habilidades (vertiente cognitiva) y disposiciones (vertiente afectiva), así como el trabajo de estrategias metacognitivas y la evaluación epistemológica (Kuhn y Weinstock, 2002) que nos permiten relacionar el aprendizaje con las operaciones, los procesos mentales y el modo de utilizarlos para lograr el objetivo planteado. El trabajo de estos elementos tiene claras implicaciones en la enseñanza (Nieto y Saiz, 2011).

Aunque el pensamiento crítico parece guardar una relación evidente con el mundo de las ciencias, la existencia de estudios que relacionan la educación musical y el pensamiento crítico han contribuido a la mejora de la comprensión de la estructura de la música, del significado de los sonidos, del valor de las composiciones y de la comprensión y contextualización histórica y social de la música (Small, 1987) proporcionando nuevos significados al aprendizaje de la música en el contexto escolar. De acuerdo con McPeck (cit. en Green, 2005:7) el pensamiento crítico es una actividad reflexiva orientada hacia la acción en un contexto de estudio, por lo que no admite la generalización.

Además del desarrollo de habilidades musicales, la relación entre la educación musical y el pensamiento crítico ha sido motivo de investigación en el estudio de la evolución de competencias matemáticas y lingüísticas (Zellner, 2011), el trabajo de destrezas personales como la resolución de problemas en la percepción auditiva (Bamberger y Brody, 1984), la motivación individual y su influencia en la actitud académica (Wallner, 2007), la implicación de la dimensión afectiva (Pogonowski, 1987, 1989), la estimulación de competencias expresivas, las relaciones interpersonales y sociales y su repercusión en la creación de entornos de aprendizaje y en la promoción de la educación intercultural (Priest, 1997; Cabedo y Díaz, 2016) entre otras.

\section{Consideraciones a nuestro estudio}

Desde una perspectiva educativa, la profundización en la praxis en el aula de música de Educación Primaria nos lleva al estudio de la relación entre: la naturaleza de la acción didáctica musical, su influencia en el modo de participación del alumno y las habilidades implicadas en la construcción del aprendizaje. El proceso educativo musical debe compaginar la educación de las habilidades auditivas, vocales, corporales, técnicas e instrumentales y 
el correspondiente razonamiento para llegar a la teorización. La significatividad del aprendizaje requiere la creación de entornos educativos que promuevan la participación, la reflexión y el disfrute del alumno en la construcción del conocimiento musical, mediante el establecimiento de conexiones entre la práctica y la teoría, como mecanismos que facilitan su comprensión.

Figura 1. Implicaciones educativas en la praxis docente

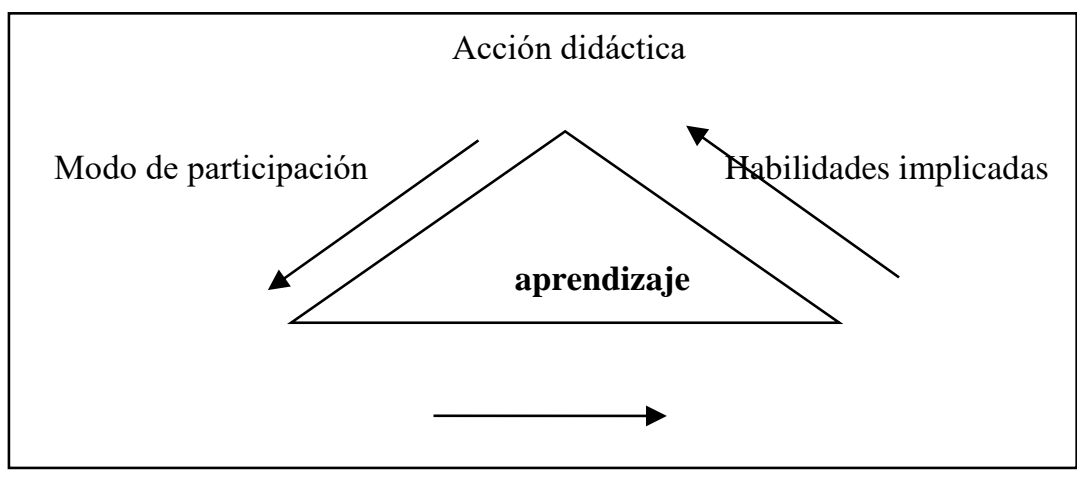

Autor: Luis del Barrio

Aunque la mayoría de las investigaciones educativas sobre el pensamiento crítico están asociadas a experiencias con estudiantes adolescentes y adultos, nuestro estudio con un grupo de Educación Primaria tiene como finalidad motivar a los niños a desarrollar su iniciativa y autonomía, su capacidad creativa y de innovación y su habilidad para pensar de manera constructiva y crítica en el aula y fuera de ella. De acuerdo con Mora (2013) la educación del pensamiento crítico y creativo constituye un verdadero reto pedagógico en el contexto de la educación básica, por el cual el niño construye el aprendizaje cuando alcanza un conocimiento crítico sobre algo desconocido y logra incorporarlo como un nuevo constructo en su bagaje experiencial.

\section{Objetivos de la investigación}

Nuestro propósito de investigación educativa tiene como finalidad estudiar el modo en el que el pensamiento crítico y creativo musical afectan en el desarrollo del aprendizaje del alumno de Educación Primaria en una experiencia colaborativa. Este propósito se concreta en los siguientes objetivos de investigación:

Objetivo 1. Analizar la evolución de las habilidades cognitivas y afectivas que estimulan el pensamiento reflexivo y crítico en el aprendizaje musical y en la educación personal del alumno. 
Objetivo 2. Identificar la evolución de las habilidades comunicativas y sociales estimuladas por la aplicación del pensamiento reflexivo y crítico.

Objetivo 3. Estudiar las repercusiones que proporciona la creación de un entorno educativo creativo y crítico en el aprendizaje musical y en el proceso educativo.

Objetivo 4. Reflexionar sobre las debilidades o desventajas que conllevan la consideración del pensamiento crítico y sus consecuencias educativas.

Objetivo 5. Identificar y valorar las contribuciones que sugieren la consideración del pensamiento crítico en la mejora del trabajo de los alumnos en el aula y en el desarrollo de su autonomía.

Objetivo 6. Analizar y valorar las exigencias profesionales que conllevan al docente la aplicación de estas estrategias didácticas en el proceso de enseñanza-aprendizaje musical.

\section{Metodología}

La profundización y la interpretación de los hechos en el ámbito de la investigación educativa contribuyen a la comprensión de los procesos formativos y al desarrollo educativo de los alumnos. Nuestro trabajo se corresponde con un estudio ideográfico fundamentado en el conocimiento individual, en la descripción de los acontecimientos y en el análisis de las habilidades cognitivas y organizativas relacionadas con el pensamiento crítico y creativo.

El estudio se basa en el desarrollo de una experiencia creativa y colaborativa musical con dos grupos clase de tercer ciclo de Educación Primaria desarrollada durante diez sesiones en el primer trimestre del curso 2017/2018 en un colegio público de la zona de escolarización 6 de Zaragoza (España). Por su naturaleza, se trata de un trabajo empírico, cuya perspectiva de investigación se basa en la fenomenografía (Marton, 1986) en la medida que trata de describir y analizar el modo en el que el alumno construye el aprendizaje desde una perspectiva práctica, reflexiva, creativa, colaborativa y crítica en un contexto educativo normalizado, dado que el estudio lo emprende el propio docente musical de los dos grupos de estudio que ejerce como maestro e investigador. El diseño de investigación cuasiexperimental tiene como finalidad estudiar la evolución cognitiva, organizativa, afectiva y social del alumno en la actividad crítica y creativa que describen la metodología didáctica de esta experiencia.

\section{Método de investigación}

La etnografía como principal método de investigación nos ha facilitado la descripción del grupo de estudio y la interpretación del conocimiento relacionado con la variable (pensamiento crítico), cuyo estudio 
y seguimiento prolongados han contribuido a su comprensión. El conocimiento obtenido en el trabajo de campo ha potenciado la transformación y la mejora de la realidad educativa de estudio (Hamersley y Atkinson, 2005) relacionada en nuestro caso con el análisis de nuevos modos de acceso, selección y gestión de la información para la construcción del aprendizaje musical. El trabajo en escenarios naturales nos ha aproximado a la realidad escolar determinada por la comprensión del entorno físico y social de los comportamientos y las interacciones de los alumnos del grupo objeto de estudio en situaciones cotidianas de aula. Por su parte, de acuerdo con Nolla Cao (1997), el autoconocimiento generado en escenarios naturales de estudio nos transforma como personas y repercute tanto en nuestro rol docente e investigador como en el aprendizaje de los alumnos haciendo de éste un estudio sobre educación, para la educación.

Respecto a las técnicas de investigación utilizamos la observación participante como principal técnica de recogida de información observando y registrando los datos más relevantes en el escenario de estudio procurando provocar la menor interferencia. La descripción reflexiva y holista y la participación durante un tiempo prolongado proporciona fiabilidad en la recogida de la información y facilita la familiarización del alumno con un nuevo modo de acción e intervención en el proceso educativo.

Durante el trabajo de campo se suceden las entrevistas etnográficas como momentos de estimulación de la reflexión del alumno, en torno a pistas de indagación relacionadas con el proceso educativo. Para el registro de la información significativa utilizamos las notas, el diario de campo, la grabación de las sesiones en video con el fin de analizar el trabajo en ellas y un cuestionario de valoración final al término del estudio.

Diseñamos y aplicamos una escala de observación de manera semanal y una hoja de autorregistro al final del estudio como instrumentos de evaluación de las disposiciones de pensamiento de las siete subescalas que recoge el California Critical Thinking Disposition Inventory (CCTDI), un instrumento de referencia para la medición de las habilidades del pensamiento crítico desarrollado por Facione y Facione (1992). Aunque éste es un instrumento aplicado con adolescentes y población adulta evaluamos las habilidades que lo identifican: búsqueda de la verdad, mentalidad abierta, capacidad de análisis, disposición para la sistematización, autoconfianza en el razonamiento, curiosidad y madurez cognitiva, de acuerdo con los indicadores relacionados con nuestro trabajo. 
Figura 2. Instrumentos de evaluación

\begin{tabular}{|c|c|c|}
\hline \multirow[t]{2}{*}{ Habilidad } & \multicolumn{2}{|c|}{ Instrumentos } \\
\hline & Hoja de autorregistro & Escala de observación \\
\hline \multirow{5}{*}{ Verdad } & $\begin{array}{l}\text { Cuando algo me interesa busco } \\
\text { información para aprender más. }\end{array}$ & $\begin{array}{c}\text { Muestra interés e inquietud por } \\
\text { aprender más. }\end{array}$ \\
\hline & $\begin{array}{l}\text { Utilizo diferentes fuentes para comprobar } \\
\text { y mejorar la información. }\end{array}$ & $\begin{array}{c}\text { Usa varias fuentes para comprobar y } \\
\text { mejorar la información. }\end{array}$ \\
\hline & Pregunto cuando necesito saber algo. & Pregunta cuando necesita saber. \\
\hline & $\begin{array}{l}\text { Modifico la información cuando } \\
\text { compruebo que es mejorable. }\end{array}$ & $\begin{array}{l}\text { Modifica la información para mejorar } \\
\text { su argumento y su trabajo. }\end{array}$ \\
\hline & Me gusta conseguir la perfección. & Es perfeccionista. \\
\hline \multirow{4}{*}{$\begin{array}{l}\text { Mentalidad } \\
\text { abierta }\end{array}$} & Siempre o casi siempre tengo razón. & Le cuesta admitir otras opiniones. \\
\hline & Me gusta escuchar a los demás & $\begin{array}{l}\text { Escucha las valoraciones de los } \\
\text { compañeros. }\end{array}$ \\
\hline & $\begin{array}{c}\text { Valoro la mejor idea en el grupo aunque } \\
\text { no sea la mía. }\end{array}$ & Reconoce la mejor idea en el grupo. \\
\hline & $\begin{array}{l}\text { Me interesa aprender nuevos modos de } \\
\text { pensar y trabajar. }\end{array}$ & $\begin{array}{l}\text { Muestra interés por practicar nuevos } \\
\text { modos de pensar y trabajar. }\end{array}$ \\
\hline \multirow{5}{*}{$\begin{array}{l}\text { Capacidad de } \\
\text { análisis }\end{array}$} & Hago planes y los cumplo. & $\begin{array}{l}\text { Planifica un orden de trabajo y lo } \\
\text { cumple. }\end{array}$ \\
\hline & Pongo atención en los detalles. & $\begin{array}{c}\text { Trabaja en la información y en los } \\
\text { detalles. }\end{array}$ \\
\hline & Prefiero las tareas que los proyectos. & Prefiere las tareas a los proyectos. \\
\hline & Me cuesta ponerme a trabajar. & Le cuesta ponerse a trabajar. \\
\hline & $\begin{array}{c}\text { Prefiero un trabajo de poco esfuerzo, que } \\
\text { uno exigente. }\end{array}$ & $\begin{array}{l}\text { Se decanta por las tareas sencillas que } \\
\text { por las que suponen esfuerzo. }\end{array}$ \\
\hline \multirow{5}{*}{ Sistematiza } & Me gusta el orden. & $\begin{array}{l}\text { Es ordenado. } \\
\end{array}$ \\
\hline & Suelo trabajar siguiendo un plan. & Sigue el plan marcado. \\
\hline & Realizo mis tareas en poco tiempo. & Busca rápida solución. \\
\hline & Relaciono lo nuevo con lo que ya sé. & Conecta los aprendizajes y pregunta \\
\hline & $\begin{array}{l}\text { Prefiero adaptar mi esfuerzo al momento } \\
\text { que seguir un plan. }\end{array}$ & No sigue un plan de trabajo. \\
\hline \multirow{5}{*}{ Confianza } & Estoy preparado. & Se siente seguro. \\
\hline & Me esfuerzo para sentirme seguro. & Se esfuerza para sentirse seguro. \\
\hline & $\begin{array}{l}\text { Confiar en trabajar el pensamiento para } \\
\text { lograr el objetivo me gusta. }\end{array}$ & Muestra interés por mejorar. \\
\hline & Me gusta pensar antes de actuar. & $\begin{array}{l}\text { Es reflexivo. } \\
\end{array}$ \\
\hline & Pensar no me resulta divertido. & Es impulsivo o no reflexivo. \\
\hline \multirow{6}{*}{ Curiosidad } & Me gusta pensar en nuevos proyectos. & Es creativo y emprendedor. \\
\hline & Prefiero problemas ingeniosos. & Le atraen los retos. \\
\hline & Comparto mis inquietudes. & Comparte sus inquietudes. \\
\hline & Busco información en varios medios. & Busca información en varios medios. \\
\hline & Incluyo aprendizajes en mis proyectos. & Relaciona ideas y conocimientos. \\
\hline & Aplico lo aprendido en otros contextos. & $\begin{array}{l}\text { Aplica lo aprendido en otros } \\
\text { contextos. }\end{array}$ \\
\hline \multirow[b]{2}{*}{$\begin{array}{l}\text { Maduración } \\
\text { cognitiva }\end{array}$} & Prefiero tareas complicadas. & Le atraen las tareas complicadas. \\
\hline & $\begin{array}{l}\text { Me gusta tener responsabilidad de } \\
\text { manejar situaciones exigentes. }\end{array}$ & $\begin{array}{c}\text { Asume con seguridad } \\
\text { responsabilidades en el desarrollo de }\end{array}$ \\
\hline
\end{tabular}




\begin{tabular}{|c|c|c|}
\hline & & tareas. \\
\cline { 2 - 3 } & $\begin{array}{c}\text { Prefiero las tareas de pensamiento } \\
\text { abstracto. }\end{array}$ & $\begin{array}{c}\text { Le atraen las tareas de pensamiento } \\
\text { abstracto }\end{array}$ \\
\cline { 2 - 3 } & $\begin{array}{c}\text { Me adapto a situaciones de distintos } \\
\text { niveles de exigencia. }\end{array}$ & $\begin{array}{c}\text { Sabe adaptarse a situaciones de } \\
\text { distintos niveles de exigencia. }\end{array}$ \\
\hline
\end{tabular}

Autor: Luis del Barrio

\section{Procedimiento}

Nuestro propósito por el estudio del pensamiento crítico nos motiva para emprender esta experiencia fundamentada en la creación de nuevos entornos educativos de promoción de la reflexión, la autonomía, la comunicación y el trabajo cooperativo entre los alumnos como medio de acceso y desarrollo del conocimiento. Para ello, proponemos la organización de cada grupo-clase en cinco grupos colaborativos de trabajo de cuatro miembros cada uno.

La pregunta que guía el tema de estudio es: ¿Conoces las obras más famosas de la música clásica? Cada grupo elige una pieza musical clásica conocida y a partir de su estudio organizamos el proceso educativo en torno a dos macrotareas. La primera es una tarea relacionada con la elaboración de infografías referidas al estudio de la obra, el compositor, el estilo y la época. La búsqueda, el análisis, la selección, la discusión, la organización de la información, así como el modo de presentación y la exposición son actividades que pretenden familiarizar a los niños con el manejo de fuentes de información y herramientas tecnológicas en torno a los que elaborar un marco de conocimiento. La segunda tarea es una tarea creativa que consiste en el diseño, la preparación y la interpretación de una actividad musical práctica en torno a la pieza musical. Cada grupo establece un objetivo musical y un modo de trabajarlo en alguno de los medios de expresión corporal, vocal o instrumental.

Para favorecer la participación y animar la iniciativa y el interés del alumno en esta nueva metodología de trabajo basada en el desarrollo de la capacidad de aprender, pensar y examinar el pensamiento, incluimos el uso de técnicas creativas como el brainstorming, el brainwriting, el SCAMPER o los mapas mentales como herramientas didácticas que estimulan la propuesta de ideas. La reflexión y la elección de las mejores ideas favorecen la construcción del conocimiento, de acuerdo con los propósitos del grupo. Por su parte, la creatividad y el diálogo son actividades naturales y sistemáticas, transformadas en estrategias didácticas que potencian el pensamiento crítico, la relación social y la actitud del alumno en un entorno de aprendizaje colaborativo.

La educación del pensamiento crítico implica un trabajo de la flexibilidad cognitiva, para admitir los cambios que puedan darse, mediante el entrenamiento de operaciones mentales como proponer, eliminar, 
combinar, sustituir, modificar, adaptar o reorganizar la información. Pensar críticamente ha conllevado salir de la propia subjetividad para escuchar los argumentos de los demás, analizar y evaluar de manera objetiva, con el fin de cambiar y mejorar el aprendizaje, y así transformar las condiciones de su entorno de acción. En este sentido, la promoción de una conducta prosocial ha requerido la consideración de la maduración sociocognitiva de los alumnos, la apertura a la socialización y al desarrollo del aprendizaje, mediante la interacción con los compañeros y el maestro. Así pues, nuestro estudio trata de motivar la inquietud del alumno para desarrollar el hábito de pensar críticamente y crear conocimiento, como actividades que favorecen una actitud educativa de emancipación.

\section{Resultados}

\section{Muestra}

Emprendemos nuestro estudio con dos grupos clase de sexto nivel de Educación Primaria del CEIP JUAN XXIII de Zaragoza (España) formados por 20 y 21 estudiantes respectivamente $(\mathrm{N}=41)$, cuyas características de género y edad son las siguientes:

Cuadro 1. Participantes en la investigación

\begin{tabular}{|c|c|c|}
\hline Componentes & Frecuencia & Porcentaje \\
\hline Niñas & 22 & 53,6 \\
\hline Niños & 19 & 46,4 \\
\hline TOTAL & 41 & 100 \\
\hline
\end{tabular}

Fuente: Secretaría de CEIP Juan XXIII

Autor: Luis del Barrio

Cuadro 2. Análisis de la edad de los participantes

\begin{tabular}{|c|c|c|}
\hline Edad/años & Frecuencia & Porcentaje \\
\hline 10 & 4 & 9,7 \\
\hline 11 & 29 & 70,7 \\
\hline 12 & 8 & 19,5 \\
\hline TOTAL & 41 & 100 \\
\hline
\end{tabular}

Fuente: Secretaría de CEIP Juan XXIII

Autor: Luis del Barrio

De acuerdo con el Cuadro 2, los participantes con una edad de 12 años llevan un curso más de escolarización que el resto, debido a la no promoción en algún curso a lo largo de la etapa de Primaria, mientras que los niños de 10 años, todavía no habían cumplido los 11 en el momento de la realización de la investigación.

\section{Análisis de resultados}

Según los datos obtenidos recogidos en la Figura 3, el desarrollo del proceso educativo sobre el estudio de un tema de interés seleccionado por los 
alumnos es el motivo principal del $63,4 \%$ de los participantes que les lleva a pensar, buscar información, contrastar fuentes y adquirir una actitud rigurosa en la búsqueda de la verdad, por encima de otros motivos.

La estimulación del pensamiento crítico en el proceso educativo ha potenciado que el 90,2 \% desarrolle una actitud indagatoria y de esfuerzo personal para tratar de alcanzar la información más fiable. Si bien es cierto que el objetivo de trabajar para lograr la perfección se corresponde con una conducta personal de alta exigencia reconocida por tan solo el $17 \%$ de los estudiantes, hemos de decir que la influencia y la responsabilidad del grupo han potenciado la implicación creativa y crítica de sus participantes en un proyecto de trabajo común.

Figura 3. Factores que inicialmente estimulan el pensamiento crítico

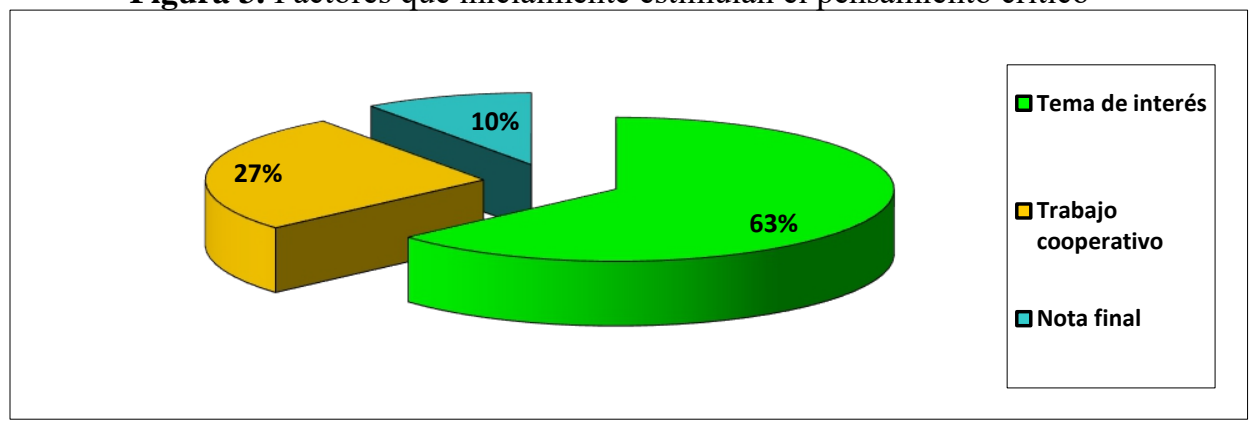

Autor: Luis del Barrio

Respecto al análisis de la habilidad mente abierta, el interés por escuchar las ideas de los demás, valorado de manera muy positiva por el $92,6 \%$ de los participantes, y el reconocimiento y la valoración de la mejor idea en el grupo demuestran la importancia atribuida al trabajo de actitudes como el respeto y la tolerancia, como aspectos clave en el desarrollo del pensamiento crítico. Por su parte, la inquietud del $70,7 \%$ de los participantes por el conocimiento de nuevos modos de pensar y trabajar evidencia una actitud flexible y emprendedora para asumir nuevos retos de aprendizaje y adaptarse a las condiciones que los determinan.

Figura 4. Interés por escuchar y valorar las ideas de los demás

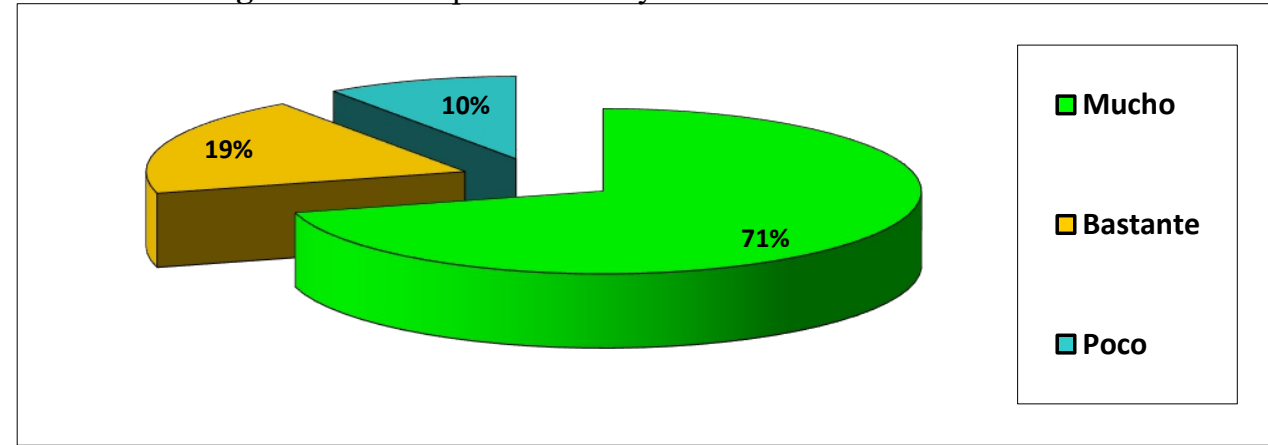

Autor: Luis del Barrio 
La valoración de la capacidad analítica nos muestra que el 80,4\% de los estudiantes dedican tiempo y esfuerzo para mejorar su trabajo y, como consecuencia, muestran una mayor seguridad, persistencia y decisión para llegar a cumplir sus objetivos respecto a quienes no lo hacen de este modo. Además de ello, el $63,4 \%$ de los participantes prefieren la realización de proyectos a las tareas sencillas, asumiendo la dificultad como un elemento atractivo considerado como reto en el proceso educativo. Los niños que poseen esta capacidad analítica y crítica han ejercido como líderes en diferentes momentos del proyecto grupal. Su contribución ha sido determinante en la orientación del trabajo y esta función les ha permitido ganarse la confianza y el respeto de sus compañeros:

- "Como la segunda parte de la melodía es muy difícil para tocar, ¿Qué os parece si tocamos la primera parte y la segunda inventamos una canción para acompañarla con otros instrumentos de percusión con ritmo como por ejemplo los bongos?".

Figura 5. Tiempo y esfuerzo dedicado por los miembros del grupo para mejorar sus trabajos

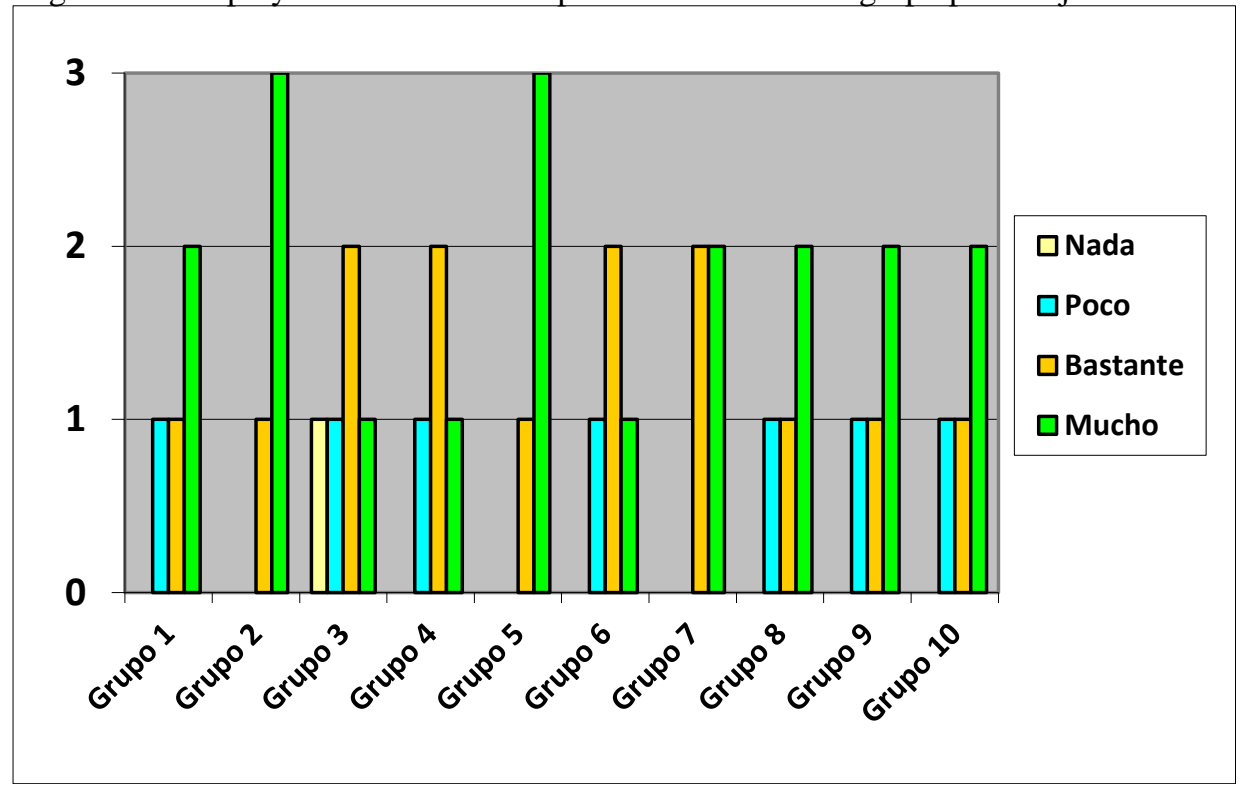

Autor: Luis del Barrio

Los estudiantes que demuestran una capacidad analítica óptima también poseen una sistematicidad a la hora de trabajar, materializada en la creación de normas para tomar decisiones: - ¿Quién vota porque incluyamos esta información?" y en el reparto de tareas, cuya confianza estimula la autonomía y el esfuerzo de los miembros del grupo para ejecutarlas. Además, el 75,6\% de los participantes construyen el aprendizaje siguiendo el plan de trabajo trazado por el grupo para cada una de las actividades del proyecto, adaptándolo a la evolución del conocimiento. La iniciativa 
personal motiva al niño a sugerir ideas, estudiar las aportaciones de los compañeros, conectar los nuevos aprendizajes con los ya adquiridos y organizar los argumentos en la construcción del aprendizaje y en la creación de entornos de aprendizaje metacognitivo.

La confianza desarrollada por los estudiantes a lo largo del estudio influye en el análisis del pensamiento crítico. El hecho de fomentar el pensamiento en el aula y plantear tareas creativas estimula la práctica de la reflexión dentro y fuera del aula como habilidades de preparación que preceden a la acción. De acuerdo con los resultados de la Figura 6 observamos cómo la curva de evolución del aspecto Me siento preparado crece semanalmente de un modo directamente proporcional a la capacidad de esfuerzo para sentirse seguro. En este sentido, a medida que el alumno se esfuerza para reconocer la utilidad de las ideas propuestas, para distribuir las categorías informativas en la infografía o para lograr una correcta interpretación instrumental o corporal, adquiere seguridad y aumenta su confianza y autonomía hasta tal punto que en las dos últimas semanas el nivel de autonomía es mayor que el nivel de esfuerzo. Este momento se corresponde con el ensayo de la tarea creativa musical. Por su parte, el planteamiento de ideas, el análisis grupal de las mismas y la toma de decisiones han estimulado la capacidad reflexiva, creativa y crítica proporcionando al alumno el gusto por pensar antes de actuar.

Figura 6. Evaluación de la confianza a lo largo del estudio

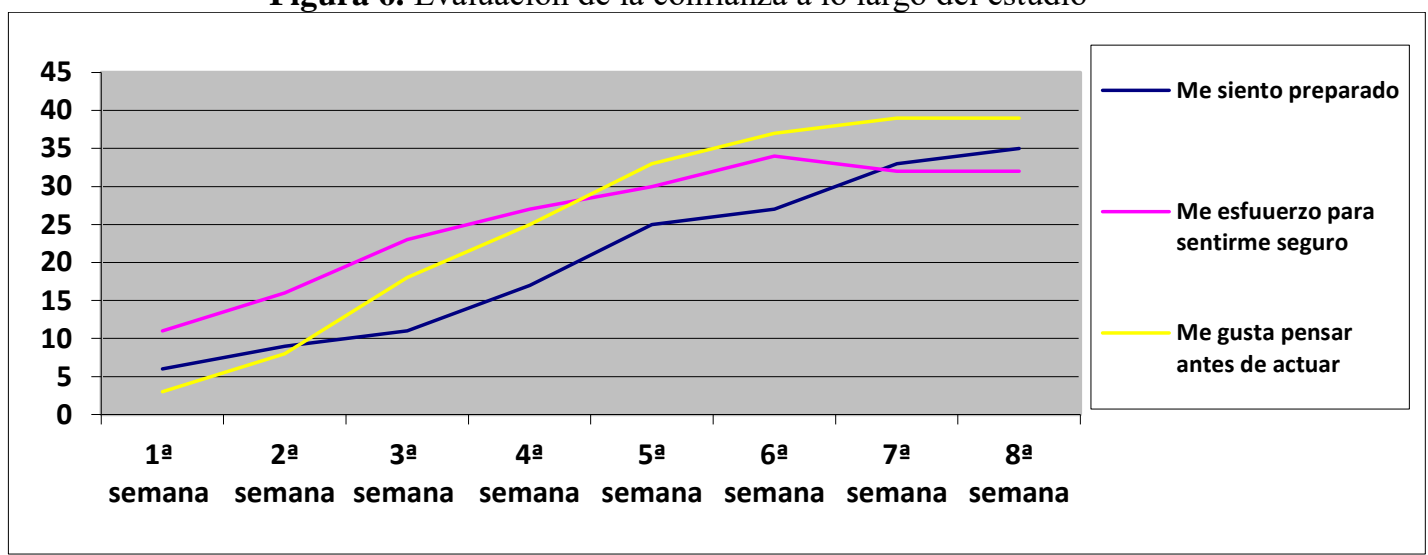

Autor: Luis del Barrio

Evocar la curiosidad es uno de los factores clave para despertar el interés del niño por el aprendizaje y la reflexión sobre el mismo. En el desarrollo del aprendizaje, el $87,8 \%$ de los estudiantes comparten sus inquietudes y sus fuentes de información con sus compañeros y el $78 \%$ busca datos en diferentes medios para fortalecer sus ideas y argumentos. La curiosidad que despierta el tema de estudio está relacionada con la utilidad 
de sus ideas para incorporarlas en su trabajo y para aplicarlas en contextos diferentes, tal y como han declarado el $65,8 \%$ de los estudiantes.

Por último, la evaluación de la maduración cognitiva nos demuestra que el $43,9 \%$ de los estudiantes prefiere realizar tareas complejas y creativas que sencillas y directivas. Tanto la infografía como la tarea creativa requieren la autonomía del estudiante para buscar, seleccionar, organizar la información y plasmarla en un trabajo artístico plástico y musical. Los grupos de trabajo heterogéneos facilitan la implicación de todos los miembros. La reflexión, el diálogo y la creatividad en el trabajo cooperativo provocan que el $78 \%$ de los participantes reconozcan asumir tareas de responsabilidad y liderazgo en algún momento del trabajo como la coordinación del grupo, el reparto de tareas, la resolución de conflictos y la dinamización en la toma de decisiones relacionadas con la elaboración del producto final.

Figura 7. Maduración cognitiva (tareas de responsabilidad)

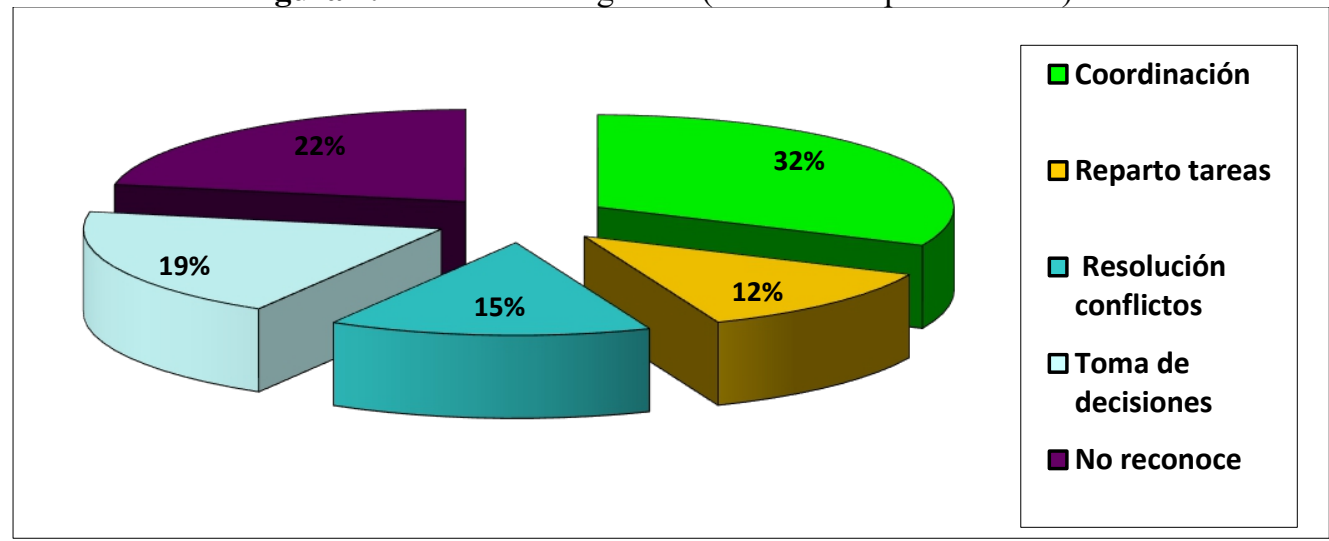

Autor: Luis del Barrio

Asimismo, el 85,3\% afirman que el trabajo cooperativo potencia su capacidad comunicativa y de colaboración y mejora su habilidad para adaptarse a los niveles de exigencia de las tareas del proyecto y a los ritmos de trabajo marcados por el propio grupo.

\section{Conclusión}

La estimulación del interés y el deseo de aprender tiene lugar cuando el niño selecciona un tema de estudio que le fascina y es significativo para él. Este hecho activa la atención y la implicación por adquirir, dominar y compartir el conocimiento. La construcción del conocimiento y la evaluación de su utilidad son operaciones mentales del pensamiento crítico trabajadas en la elaboración del producto creativo artístico y musical. El aprendizaje cooperativo motiva la implicación personal y la puesta en común de las habilidades individuales en la elaboración de un proyecto colectivo. El trabajo creativo en grupo potencia la habilidad de pensar, proponer, 
escuchar, contrastar y tomar decisiones sobre la construcción del aprendizaje en un entorno educativo crítico y colaborativo. El carácter novedoso de esta experiencia y la autorrealización determinan la autoestima, la responsabilidad y la autonomía emocional en el emprendimiento de las tareas educativas.

Respecto a las debilidades del pensamiento crítico, los estudiantes que destacan por una predominante dimensión crítica del pensamiento muestran una conducta de cuestionamiento habitual que, en ocasiones, afecta al consenso en la toma de decisiones y al ritmo de trabajo del grupo.

La promoción del pensamiento crítico en el proceso educativo musical en Educación Primaria integra la habilidad de pensar como una actividad natural de autoaprendizaje que estimula la comunicación y la reflexión como actitudes de acceso y análisis del conocimiento desde las que se promueve la educación de la autonomía y la madurez del estudiante. El docente como director del proceso educativo debe clarificar los objetivos del trabajo, promover el pensamiento y el diálogo en el aula, fomentar la búsqueda de la verdad, la escucha activa y la flexibilidad, facilitar herramientas metodológicas y de evaluación del aprendizaje que permitan analizar su aplicabilidad y reconocer y potenciar los valores que ayudan a la educación emancipatoria del alumno.

\section{References:}

1. Bamberger, J. y Brody, M. (1984). Perceptual problema solving in music: some proposals for future research, en Psychomusicology, 4, (1-2), pp. 33-58.

2. Cabedo-Mas, A. y Díaz-Gómez, M. (2016). Music Education for the Improvement of Coexistence in an beyond the Classroom: A Study Based on the Consultation of Experts, en Teachers and Teaching: Theory and Practice, 22, (3), pp. 368-386.

3. Ennis, R. H. (1985). A logical basis for measuring critical thinking skills, en Educational Leadership, 43, (2), pp. 44-48.

4. Ennis, R. H. (2011). The nature of critical thinking: An outline of critical thinking dispositions and abilities. Presetnation at the Sixth International Conference on Thinking at MIT, Cambridge, MA, July, 1994. Last revised May, 2011. Recuperado de http://faculty.education.illinois.edu/rhennis/documents/TheNatureofC riticalThinking_51711_000.pdf

5. Facione, P.; Facione, N. (1992). The California Critical Thinking Dispositions Inventory (CCTDI); and the CCTDI Test manual. Millbrae, CA: The California Academic Press, 
6. Green, M. C. (2005). Factors influencing the process of critical thinking among health professional during computer conferencing: a case study. Unpublished master thesis. Athabasca University.

7. Guzmán, S. y Sánchez-Escobedo, P. (2006). Efectos de un programa de capacitación de profesores en el desarrollo de habilidades de pensamiento crítico en estudiantes universitarios en el sureste de México, en Revista Electrónica de Investigación Educativa, 8(2).

8. Halpern, D. (1998). Critical thinking, en American Psychologist, 53(4), pp. 449-455.

9. Hammersley, M. y Atkinson, P. (2005). Etnografía. Métodos de investigación. Barcelona: Paidós.

10. Jones, B, F., y Idol, L. (1990). Introduction, en B. F. Jones y L. Idol (Eds.), Dimensions of thinking and cognitive instruction pp.1-13. Hillsdale: NJ, Erlbaum.

11. Kuhn, D. y Weinstock, M. (2002). What is epistemological thinking and why does it matter?, in B.K. Hofer, and P.R. Pintrich (eds.), Personal epistemology: The psychology of beliefs about knowledge and knowing. Mahwah, N.J.: Erlbaum.

12. Lipman, M. (1998). Pensamiento complejo y educación. Madrid: De la Torre.

13. Marton, F. (1986). Phenomenography: A research approach investigating different understandings of reality, en Journal of Thought, 21, pp. 28-49.

14. Mertes, L. (1991). Thinking and writing, en Middle School Journal, 22, pp. 24-25.

15. Mora, F. (2013). Neuroeducación. Madrid: Alianza Editorial.

16. Nieto, A. M. y Saiz, C. (2011). Skills and dispositions of gritical thinking: are they sufficient? en Anales de Psicología, 27 (1), pp. 202-209.

17. Nolla Cao, N. (1997). Etnografía: una alternativa más en la investigación pedagógica, Revista Cubana de Educación Media y Superior, 11, pp. 107-115.

18. Pogonowski, L. (1987). Developing skills in critical thinking and problem solving, en Music Educators Journal, 73, (6), pp. 37-41.

19. Pogonowski, L. (1989). Critical thinking and music listening, en Music Educators Journal, 76, (1), pp. 35-38.

20. Priest, L.T. (1997). Fostering creative and critical thinking in a beginning instrumental music class. Unpublished doctoral dissertation. University of Illinois.

21. Saiz, C. y Rivas, S.F. (2008). Intervenir para transferir en pensamiento crítico, en. Praxis, 10 (13), pp. 129-149. 
22. Small, A. R. (1987). Music and critical thinking: what do we need to know? in Music Educators Journal, 74, (1), pp. 46-49.

23. UNESCO (1998). La educación superior en el s. XXI: visión y acción, en Conferencia Mundial sobre la Educación Superior. París.

24. Wallner, G. D. (2007). The impact of music education on academic achievement, attendance rate, and student conduct on the 2006 senior class in one southeast Virginia public school division. Unpublished doctoral dissertation. Blacksburg University.

25. Zellner, R. M. (2011). A study of the relationship between instrumental music education and critical thinking in 8th-and 11thgrade students. Unpublished doctoral dissertation Capella University 